\title{
Applications of synthetic gene networks
}

\section{FUQING WU and XIAO WANG}

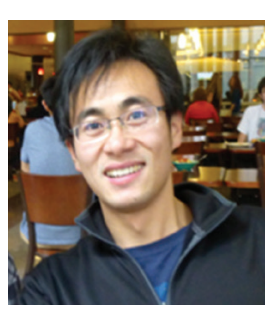

Fuqing Wu received his Master Degree in the Wuhan Institute of Virology, Chinese Academy of Sciences. He is currently pursuing his $\mathrm{PhD}$ in Biomedical Engineering at Arizona State University, Tempe, AZ 85287, USA. His research interests focus on using synthetic biology approaches to engineer genetic circuits to carry out a wide range of functions. Particularly, he is interested in combining mathematical modelling with experimental validations to reveal the fundamental mechanisms generating multistability and state transition dynamics during stem cell differentiation. He can be contacted by E-mail at fuqingwu@asu.edu.

Xiao Wang is an assistant professor in Biomedical Engineering at Arizona State University. He received his $\mathrm{PhD}$ at the University of North Carolina at Chapel Hill in 2006. As the Principal Investigator of the Systems and Synthetic Biology Research Group, he is interested in using both forward (synthetic biology) and reverse (systems biology) engineering approaches to understand biology. Specific research topics include engineering synthetic multistable gene networks, systems

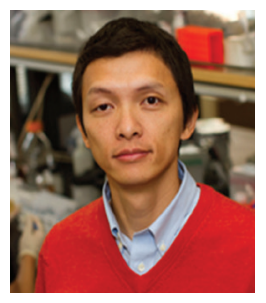
biology research on small network motifs with feedbacks, understanding the role of noise in cell differentiation and development, and analysing molecular evolution. He can be contacted by E-mail at xiaowang@asu.edu.

\begin{abstract}
Synthetic gene networks have evolved from simple proof-of-concept circuits to complex therapy-oriented networks over the past 15 years. This advancement has greatly facilitated the expansion of the emerging field of synthetic biology. In this review, we highlight the main applications of synthetic gene networks in understanding biological design principles, developing biosensors for diagnosis, producing industrial and biomedical compounds, and treating human diseases. Finally, we outline current challenges and future prospects of synthetic gene networks for advancing practical applications.
\end{abstract}

Keywords: synthetic gene networks, biological design principles

\section{Introduction}

After 15 years of rapid development, synthetic biology has started to become an engineering discipline with the aim to create, control and program cellular behaviour for basic research, and industrial and biomedical applications ${ }^{1,2}$. By taking a bottom-up approach, synthetic biologists rationally assemble biological modules such as genetic parts (promoters, coding sequence, terminators, etc.) together to create a "circuitry", which is often called a synthetic gene circuit or network, to carry out a wide range of functions. In an integrated circuitry, genes do 
not work independently but in a network. For example, the lysis-lysogeny decision of bacteriophage lambda is tightly regulated by CI-Cro genetic switch ${ }^{3}$. CI protein can repress Cro to maintain a lysogenic state, while Cro inhibits CI expression to promote lytic development.

Most existing synthetic gene networks utilise one or more of the three regulation mechanisms: transcriptional, translational, and post-translational ${ }^{4,5}$. Transcriptional circuits are engineered based on cell transcriptional regulatory machinery involving promoters, transcriptional factors, and RNA polymerase. Early examples of such synthesised circuits include the toggle switch, which is constructed from a pair of repressor genes that inhibit the transcription of each other and can be induced to flip between two stable steady states ${ }^{6}$, and the repressilator, which is composed of three repressor-promoter interactions to form a cyclic negative-feedback loop and exhibits periodic oscillating behaviour ${ }^{7}$. Since then, a myriad of transcriptional networks for diverse purposes have been constructed, including negative feedbacks ${ }^{8}$, genetic counters ${ }^{9}$, synchronised oscillators ${ }^{10}$, band-pass filters ${ }^{11}$, pulse generator ${ }^{12}$, edge detector ${ }^{13}$, and various genetic logic gates such as AND, OR, NOT, NOR, XOR, and XNOR ${ }^{14-16}$ (Table 1). Similar to electronic circuits, logic functions can also operate in living cells producing certain outputs based on environmental and cellular inputs. For example, the lac operon in Escherichia coli works as an AND gate, where $\beta$-galactosidase is only produced in the presence of both lactose and cyclic adenosine monophosphate ${ }^{17}$. This presents living cells as a conducive environment for the operation of synthesised genetic circuits designed using synthetic biology tools for conducting different applications.

Moreover, many engineered riboswitches are developed to regulate gene expression at the translational level. These RNA-based genetic switches can be easily modulated to target gene networks and reprogram cellular behaviour. For example, Green and colleagues recently engineered "Toehold switches" using non-coding RNAs to construct multi-input AND logic evaluator and regulate endogenous gene expression ${ }^{18}$. Post-translational circuits generally rely on creating novel chimeric proteins, such as protein receptors, to rewire natural signalling pathways and reshape the dynamics of signalling transduction ${ }^{4,19}$. Also, it should be noted that some hybrid synthetic circuits are engineered by integrating RNA devices into transcriptional regulatory networks ${ }^{20}$, and some synthetic gene networks are constructed and expressed in vitro ${ }^{21}$.

Starting with engineering of simple gene circuits in E. coli cells, synthetic biology has now upgraded into complex higher-order gene networks and systems with predictable functions in mammalian cells and real-world applications for human health ${ }^{1,22}$. Here, we mainly focus on the recent applications of synthetic gene networks in basic research, industry and biotherapy.

\section{2. "Build to understand"}

Whether in bacteria or in humans, gene regulatory networks of thousands of genes and interactions play critical roles in fundamental biological processes such as metabolism, signalling transduction, cell differentiation and development ${ }^{23}$. However, it is almost impossible for traditional biologists to investigate 
Table 1 Architecture of genetic logic gates and truth table

\begin{tabular}{|c|c|c|c|c|}
\hline \multirow{2}{*}{ Gate } & \multirow{2}{*}{ Architecture } & \multicolumn{3}{|c|}{ Truth Table } \\
\hline & & Signal 1 & Signal 2 & Output \\
\hline \multirow{4}{*}{ AND } & & 0 & 0 & 0 \\
\hline & & 1 & 0 & 0 \\
\hline & & 0 & 1 & 0 \\
\hline & & 1 & 1 & 1 \\
\hline \multirow{4}{*}{ OR } & & 0 & 0 & 0 \\
\hline & & 1 & 0 & 1 \\
\hline & & 0 & 1 & 1 \\
\hline & & 1 & 1 & 1 \\
\hline \multirow[t]{2}{*}{ NOT } & & \multicolumn{2}{|c|}{0} & 1 \\
\hline & & \multicolumn{2}{|c|}{1} & 0 \\
\hline \multirow{4}{*}{ NOR } & & 0 & 0 & 1 \\
\hline & & 1 & 0 & 0 \\
\hline & & 0 & 1 & 0 \\
\hline & & 1 & 1 & 0 \\
\hline \multirow{4}{*}{ XOR } & & 0 & 0 & 0 \\
\hline & & 1 & 0 & 1 \\
\hline & & 0 & 1 & 1 \\
\hline & & 1 & 1 & 0 \\
\hline \multirow{4}{*}{ XNOR } & & 0 & 0 & 1 \\
\hline & & 1 & 0 & 0 \\
\hline & & 0 & 1 & 0 \\
\hline & & 1 & 1 & 1 \\
\hline
\end{tabular}

systematically the large-scale interconnected networks in their natural context using conventional tools. Synthetic gene networks, on the other hand, provide a clear and isolated platform for uncovering the complex regulatory mechanisms, such as gene expression noise, multistability, cell decision-making, and cell-cell communications ${ }^{24,25}$. These network topologies are usually abstracted from natural analogues, built with standardised genetic modules from scratch, and investigated in living cells. Mathematical modelling as a complementary tool is also employed to understand quantitatively the network dynamics and guide experimental design.

As a milestone achievement in synthetic biology, the toggle switch, in which two genes inhibited each other, explicitly illustrated the idea $^{6}$. By using external inducers, the circuit could be controlled to toggle between two stable steady states (bistable) predicted by their mathematical model. Further studies by Wang and colleagues engineered similar bistable gene networks to reveal the mechanism for stochastic cell-fate determination in a multistable system, suggesting a new perspective on cell differentiation and development ${ }^{25}$. By combining mathematical modelling and experimental measurements, this study demonstrated that random and irreversible binary cellular state determination can be realised through initialising 
gene regulatory networks at the boundary between dynamic attractors. As another example, to gain insight into the mechanism of circadian rhythm, researchers have built several genetic clock circuits to generate synchronised oscillations in living cells and to elucidate the molecular dynamics of oscillatory behaviour ${ }^{10,26,27}$.

Furthermore, synthetic gene networks can be employed to investigate complex population interactions such as crosstalk in quorum sensing (QS), which is a widespread mechanism of bacterial communication and of biofilm formation ${ }^{28}$. Recently, Wu et al. ${ }^{29}$ found that by engineering synthetic gene networks, QS crosstalk can be dissected into signal crosstalk and promoter crosstalk. Further investigations using synthetic positive feedback circuits revealed that signal crosstalk significantly decreases the bistable potential while promoter crosstalk can be modulated to yield trimodal responses resulting from noise-induced state transitions and circuit-host interactions (Figure 1). The distinct dynamics caused by QS crosstalk indicate that we may exploit the crosstalk to artificially interfere and disrupt cell-cell communication to collapse the bacterial population's coordination, and finally treat QS-related diseases. Moreover, the concept of combining nonlinear dynamics and host-circuit interactions to modulate population dynamics broadens our understanding of mechanisms of cell-cell variability and population diversity.

Altogether, synthetic gene networks enable us to reduce biological complexity and these synthetic replicas will further help us to understand fundamental mechanisms in natural systems.

Biobricks

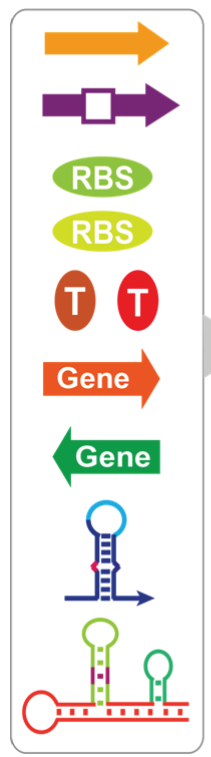

Synthetic gene networks
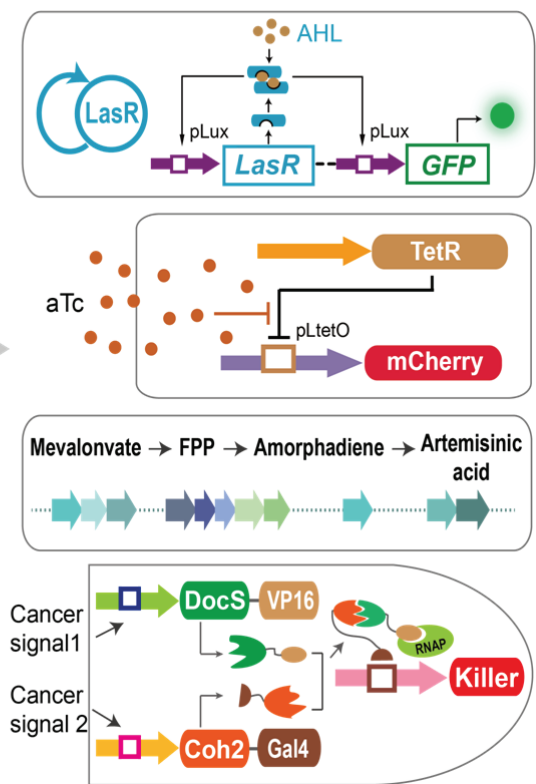

Applications
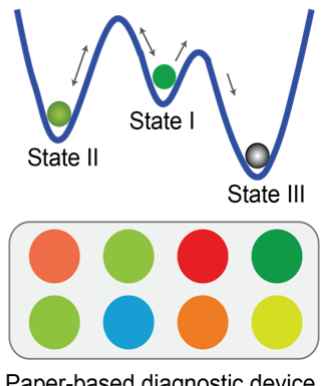

Paper-based diagnostic device
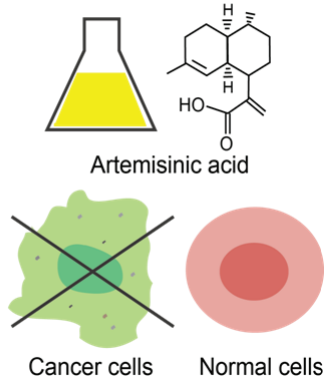

Figure 1 Assembly and applications of synthetic gene networks. From left to right: standardised biological blocks (biobricks) are employed to assemble synthetic gene networks for different applications. From top to bottom: synthetic gene network built to understand the biological mechanism ${ }^{29}$; genetic circuits for the paper-based biosensor ${ }^{32}$; biosynthetic pathway for the pharmaceutical drugs $s^{38}$; and genetic circuit for anti-cancer therap $y^{41}$. 


\section{Synthetic gene networks in single-cell organisms: biosensor, biofuel, and biopharmaceutical}

Application-oriented synthetic gene networks in single-cell organisms for solving realistic problems are also being investigated. The biosensor is one of the major applications. Generally, a biosensor is composed of two genetic parts, a sensing element responsible for recognising and interacting with the analyte of interest, and a detector element to transform the interaction signal and report it in a user-friendly way ${ }^{4}$. For example, Wang et al. ${ }^{30}$ designed a set of singleinput cellular biosensors which contain an environmental-responsible promoter and corresponding transcriptional factors, and a fluorescent output to detect the concentration of copper, mercury, zinc, and cadmium in an aqueous environment. Moreover, Kotula et al. ${ }^{31}$ engineered living bacteria with synthetic genetic circuits comprising a trigger element and a memory element to sense, record and report environmental stimuli in the mammalian gut, which lays the foundation for developing living diagnostics and therapeutics for humans. Recently, Pardee et al..$^{32}$ integrated synthetic gene networks with a paper-based technology to develop low-cost and practical paper-based biosensors to monitor the glucose and strain-specific Ebola virus (Figure 1).

In addition, engineering of biosynthetic pathways for cost-effective and highyield microbial production of interest (such as biofuels) is another important application of synthetic gene networks. Metabolic pathways of interest are often found in natural organisms but the natural yield is usually very low owing to the host's tight regulation. So a promising technique is to use a synthetic biology approach to improve the host's production capacity either by optimising the metabolic flux or by transplanting the whole biosynthetic pathway into industrial model microbes, such as E. coli and Saccharomyces cerevisiae ${ }^{4,33}$. For example, Atsumi and colleagues transferred the butanol-producing pathway from Clostridium acetobutylicum into $E$. coli and genetically modified a number of $E$. coli genes to improve 1-butanol production ${ }^{34}$. Also, S. cerevisiae has been used as a cell factory to overproduce fatty acid ethyl esters (biodiesel) by eliminating non-essential fatty acid utilisation pathways ${ }^{35}$. So far, the bioproduction of many biofuels including ethanol, butanol, biodiesel, 2-propanol, and hydrogen have been successfully explored and scaled-up through engineering of biosynthetic pathways $^{36,37}$.

Furthermore, biosynthetic pathway engineering is applied to produce rare pharmaceutical drugs. One of the successful examples is the production of the antimalarial drug artemisinin, which is a sesquiterpene endoperoxide originally produced by the plant Artemisia annua. In 2006, the Keasling group programmed $S$. cerevisiae to produce high titres of artemisinin precursor artemisinic acid by engineering of the farnesyl pyrophosphate biosynthetic pathway and introducing amorphadiene synthase and a novel cytochrome P450 monooxygenase from $A$. annua $^{38}$ (Figure 1). Recently, they further demonstrated the complete biosynthetic pathway for high-level production of artemisinic acid and developed an efficient chemical process for conversion of artemisinic acid to $\operatorname{artemisinin}^{39}$. 


\section{Therapy-oriented synthetic gene networks in mammalian cells}

The synthetic gene network is also being utilised in clinical therapy ${ }^{40}$. Using engineering synthetic gene networks as an alternative to conventional pharmacotherapy to combat human diseases is promising. The basic idea is to utilise synthetic gene circuits as a smart sensor-actuator device to interface functionally with the host's complex endogenous networks to monitor disease signals in vivo and make therapeutic responses automatically. We will now briefly consider applications of synthetic gene networks to treat cancer, diabetes, obesity, and immunotherapy.

One of the challenges for cancer therapy is to separate cancer cells from the surrounding normal cells. So the principle is to design devices that can specifically sense cancer signals such as overexpressed oncogenes and then initiate the killing process. For example, Nissim and colleagues constructed a dual-promoter integrator to target and kill cancer cells based on their cancerous context (Figure 1). Each of the two cancer cell-specific promoters drive a chimeric protein expression, which are then combined together to activate the thymidine kinase expression to kill the cell. So this AND logic circuit will be only activated in host cells expressing the two signals simultaneously ${ }^{41}$. A similar strategy was also used in a recent study in which the authors engineered a multi-input RNAi-based logic circuit to specifically identify cancer cells $\mathrm{s}^{42}$. This cancer cell classifier functions through detecting the expression levels of sets of endogenous microRNAs in HeLa cells and triggering cell apoptosis when the expression levels match a predefined profile.

Synthetic gene circuits have also been engineered for the treatment of metabolic disorders. For example, the Fussenegger group designed a synthetic optogenetic gene circuit to regulate blood-glucose homeostasis ${ }^{43}$. This circuit enables cells to respond to blue light and activate endogenous NFAT signalling (nuclear factor of activated $\mathrm{T}$ cells) to finally regulate the glucagon-like peptide 1 expression, which increases insulin levels and enhances glucose homeostasis in diabetic mice. They also developed synthetic circuits to combat diet-induced obesity in mice, hyperuricaemia and metabolic syndrome ${ }^{44-46}$.

Last but not the least, synthetic gene circuits have also been applied in immunotherapy. For example, Chen et al. engineered a synthetic ribozyme switch to regulate gene expression and T-cell proliferation in vitro and in vivo ${ }^{47}$. Wendell Lim's group developed synthetic feedback modulators and pause switches using bacterial virulence proteins to tune precisely T-cell response amplitude and control human primary CD4+ T-cell activation ${ }^{48}$.

\section{Conclusions}

We have briefly reviewed the applications of synthetic gene networks in the understanding of biological designs and mechanisms, developing biosensors for diagnosis, producing industrial and biomedical compounds, and treating human diseases. However, most of the current synthetic circuits are relatively simple both topologically and functionally. So how to construct experimentally complex and multi-node networks with predicted functions is a big challenge. For example, it is still difficult experimentally to build gene networks of fully connected triads with 
complete auto-activation (such as the Oct4-Sox2-Nanog triad) which are important to understand multistability in cell differentiation and human development ${ }^{49}$. To achieve this, more standardised and well-characterised biobricks and devices will be necessary for circuit engineering ${ }^{5}$.

Nevertheless, synthetic gene networks will accelerate the process towards practical applications, especially in the industrial and biomedical fields. So far, engineering of biosynthetic pathways using simple microbes for large-scale and cost-effective renewable energy and pharmaceuticals is still immature and awaits further development. This will rely on our complete understanding of the natural biosynthetic pathways, metabolic flux and regulatory mechanisms ${ }^{50}$. On the other hand, the overexpressed networks place a metabolic burden on the host cells, so synthetic biologists need to balance the final output and the microbes' stresses for product maximisation. It is also very encouraging that engineering of therapy-oriented gene circuits might be used to rewire the biological pathways for public health problems. Compared to traditional drugs, synthetic gene networks have unique advantages such as sensing disease states and making corresponding therapeutic decisions in a self-sufficient manner, easy engineering and manipulation, and providing sustainable protection ${ }^{51,52}$. Finally, integrating synthetic gene networks with other biomedical approaches such as cell therapy, gene therapy and biomaterials will be essential to realise the potential of synthetic biology.

\section{Acknowledgements}

Research at Dr Xiao Wang's laboratory at Arizona State University is financially supported by the National Science Foundation, the National Institute of Health, and the American Heart Association.

\section{References}

1. Cameron, D.E., Bashor, C.J. and Collins, J.J. (2014) A brief history of synthetic biology. Nat. Rev. Microbiol., 12, 381-390.

2. Elowitz, M. and Lim, W. (2010) Build life to understand it. Nature, 468, 889-890.

3. Oppenheim, A.B., Kobiler, O., Stavans, J., Court, D.L. and Adhya, S. (2005) Switches in bacteriophage lambda development. Annu. Rev. Genet., 39, 409-429.

4. Khalil, A.S. and Collins, J.J. (2010) Synthetic biology: applications come of age. Nature Rev. Genet., 11, 367-379.

5. Lu, T.K., Khalil, A.S. and Collins, J.J. (2009) Next-generation synthetic gene networks. Nat. Biotechnol., 27, $1139-1150$.

6. Gardner, T.S., Cantor, C.R. and Collins, J.J. (2000) Construction of a genetic toggle switch in Escherichia coli. Nature, 403, 339-342.

7. Elowitz, M.B. and Leibler, S. (2000) A synthetic oscillatory network of transcriptional regulators. Nature, 403, 335-338.

8. Nevozhay, D., Adams, R.M., Murphy, K.F., Josić, K. and Balázsi, G. (2009) Negative autoregulation linearizes the dose-response and suppresses the heterogeneity of gene expression. Proc. Natl. Acad. Sci., 106, $5123-5128$.

9. Friedland, A.E. et al. (2009) Synthetic gene networks that count. Science, 324, 1199-1202.

10. Danino, T., Mondragón-Palomino, O., Tsimring, L. and Hasty, J. (2010) A synchronized quorum of genetic clocks. Nature, 463, 326-330.

11. Sohka, T. et al. An externally tunable bacterial band-pass filter. (2009) Proc. Natl. Acad. Sci., 106, $10135-10140$. 
12. Basu, S., Mehreja, R., Thiberge, S., Chen, M.-T. and Weiss, R. (2004) Spatiotemporal control of gene expression with pulse-generating networks. Proc. Natl. Acad. Sci. USA, 101, 6355-6360.

13. Tabor, J.J. et al. (2009) A synthetic genetic edge detection program. Cell, 137, 1272-1281.

14. Wang, B., Kitney, R.I., Joly, N. and Buck, M. (2011) Engineering modular and orthogonal genetic logic gates for robust digital-like synthetic biology. Nat. Commun., 2, 508.

15. Siuti, P., Yazbek, J. and Lu, T.K. (2013) Synthetic circuits integrating logic and memory in living cells. Nat. Biotechnol., 31, 448-452.

16. Stanton, B.C. et al. (2014) Genomic mining of prokaryotic repressors for orthogonal logic gates. Nat. Chem. Biol., 10, 99-105.

17. Görke, B. and Stülke, J. (2008) Carbon catabolite repression in bacteria: many ways to make the most out of nutrients. Nat. Rev. Microbiol., 6, 613-624.

18. Green, A.A., Silver, P.A., Collins, J.J. and Yin, P. (2014) Toehold switches: de-novo-designed regulators of gene expression. Cell, 159, 925-939.

19. Bashor, C.J., Helman, N.C., Yan, S. and Lim, W.A. (2008) Using engineered scaffold interactions to reshape MAP kinase pathway signaling dynamics. Science, 319, 1539-1543.

20. Deans, T.L., Cantor, C.R. and Collins, J.J. (2007) A tunable genetic switch based on RNAi and repressor proteins for regulating gene expression in mammalian cells. Cell, 130, 363-372.

21. Padirac, A., Fujii, T. and Rondelez, Y. (2012) Bottom-up construction of in vitro switchable memories. Proc. Natl. Acad. Sci. USA, 109, E3212-3220.

22. Cheng, A.A. and Lu, T.K. (2012) Synthetic biology: an emerging engineering discipline. Annu. Rev. Biomed. Eng., 14, 155-178.

23. Karlebach, G. and Shamir, R. (2008) Modelling and analysis of gene regulatory networks. Nat. Rev. Mol. Cell Biol., 9, 770-780.

24. Mukherji, S. and van Oudenaarden, A. (2009) Synthetic biology: understanding biological design from synthetic circuits. Nat. Rev. Genet., 10, 859-871.

25. Wu, M. et al. (2013) Engineering of regulated stochastic cell fate determination. Proc. Natl. Acad. Sci. USA, 110, 10610-10615.

26. Stricker, J. et al. (2008) A fast, robust and tunable synthetic gene oscillator. Nature, 456, 516-519.

27. Tigges, M., Marquez-Lago, T.T., Stelling, J. and Fussenegger, M. (2009) A tunable synthetic mammalian oscillator. Nature, 457, 309-312.

28. Ng, W.-L. and Bassler, B.L. (2009) Bacterial quorum-sensing network architectures. Annu. Rev. Genet., 43, 197-222.

29. Wu, F., Menn, D.J. and Wang, X. (2014) Quorum-sensing crosstalk-driven synthetic circuits: from unimodality to trimodality. Chem. Biol., 21, 1629-1638.

30. Wang, B., Barahona, M. and Buck, M. (2013) A modular cell-based biosensor using engineered genetic logic circuits to detect and integrate multiple environmental signals. Biosens. Bioelectron., 40, $368-376$.

31. Kotula, J.W. et al. (2014) Programmable bacteria detect and record an environmental signal in the mammalian gut. Proc. Natl. Acad. Sci., 111, 4838-4843.

32. Pardee, K. et al. (2014) Paper-based synthetic gene networks. Cell, 159, 940-954.

33. Wu, M.-C., Law, B., Wilkinson, B. and Micklefield, J. (2012) Bioengineering natural product biosynthetic pathways for therapeutic applications. Curr. Opin. Biotechnol., 23, 931-940.

34. Atsumi, S. et al. (2008) Metabolic engineering of Escherichia coli for 1-butanol production. Metab. Eng., 10, 305-311.

35. Valle-Rodríguez, J.O., Shi, S., Siewers, V. and Nielsen, J. (2014) Metabolic engineering of Saccharomyces cerevisiae for production of fatty acid ethyl esters, an advanced biofuel, by eliminating non-essential fatty acid utilisation pathways. Appl. Energy, 115, 226-232.

36. Savage, D.F., Way, J. and Silver, P.A. (2008) Defossiling fuel: how synthetic biology can transform biofuel production. ACS Chem. Biol., 3, 13-16.

37. Peralta-Yahya, P.P., Zhang, F., del Cardayre, S.B. and Keasling, J.D. (2012) Microbial engineering for the production of advanced biofuels. Nature, 488, 320-328.

38. Ro, D.-K. et al. (2006) Production of the antimalarial drug precursor artemisinic acid in engineered yeast. Nature, 440, 940-943.

39. Paddon, C.J. et al. (2013) High-level semi-synthetic production of the potent antimalarial artemisinin. Nature, 496, 528-532. 
40. Ruder, W.C., Lu, T. and Collins, J.J. (2011) Synthetic biology moving into the clinic. Science, 333, $1248-1252$.

41. Nissim, L. and Bar-Ziv, R.H. (2010) A tunable dual-promoter integrator for targeting of cancer cells. Mol. Syst. Biol., 6, 444.

42. Xie, Z., Wroblewska, L., Prochazka, L., Weiss, R. and Benenson, Y. (2011) Multi-input RNAi-based logic circuit for identification of specific cancer cells. Science, 333, 1307-1311.

43. Ye, H., Baba, M.D.-E., Peng, R.-W. and Fussenegger, M. (2011) A synthetic optogenetic transcription device enhances blood-glucose homeostasis in mice. Science, 332, 1565-1568.

44. Rössger, K., Charpin-El-Hamri, G. and Fussenegger, M. (2013) A closed-loop synthetic gene circuit for the treatment of diet-induced obesity in mice. Nat. Commun., 4, 2825.

45. Ye, H. et al. (2013) Pharmaceutically controlled designer circuit for the treatment of the metabolic syndrome. Proc. Natl. Acad. Sci., 110, 141-146.

46. Kemmer, C. et al. (2010) Self-sufficient control of urate homeostasis in mice by a synthetic circuit. Nat. Biotechnol., 28, 355-360.

47. Chen, Y.Y., Jensen, M.C. and Smolke, C.D. (2010) Genetic control of mammalian T-cell proliferation with synthetic RNA regulatory systems. Proc. Natl. Acad. Sci., 107, 8531-8536.

48. Wei, P. et al. (2012) Bacterial virulence proteins as tools to rewire kinase pathways in yeast and immune cells. Nature, $\mathbf{4 8 8}, 384-388$.

49. Faucon, P.C. et al. (2014) Gene networks of fully connected triads with complete auto-activation enable multistability and stepwise stochastic transitions. PLOS ONE, 9, e102873.

50. Yadav, V.G., De Mey, M., Giaw Lim, C., Kumaran Ajikumar, P. and Stephanopoulos, G. (2012) The future of metabolic engineering and synthetic biology: towards a systematic practice. Metab. Eng., 14, $233-241$.

51. Ye, H., Aubel, D. and Fussenegger, M. (2013) Synthetic mammalian gene circuits for biomedical applications. Curr. Opin. Chem. Biol., 17, 910-917.

52. Ye, H. and Fussenegger, M. (2014) Synthetic therapeutic gene circuits in mammalian cells. FEBS Lett., 588, 2537-2544. 